\title{
Developments of the TEUFEL injector racetrack microtron
}

\author{
J.I.M. Botman ${ }^{a, *}$, J.L. Delhez a, H.L. Hagedoorn ${ }^{\text {a }}$, W.J.G.M. Kleeven ${ }^{\text {a }}$, M.H.M. Knoben ${ }^{\text {a }}$, \\ C.J. Timmermans a , G.A. Webers a, G.J. Ernst ${ }^{\text {b }}$, J.W.J. Verschuur ${ }^{\text {b }}$, W.J. Witteman ${ }^{\text {b }}$ \\ ${ }^{a}$ Eindhoven Unversity of Technology, P.O. Box 513, $5600 \mathrm{MB}$ Eindhoven, The Netherlands \\ ${ }^{b}$ Twente University, P.O. Box 217, 7500 AE Enschede, The Netherlands
}

\begin{abstract}
In this paper we report on developments of the $25 \mathrm{MeV}$ racetrack microtron (RTM) that will be the electron source for the second phase of the TEUFEL project, to generate radiation of $10 \mu \mathrm{m}$ in a $2.5 \mathrm{~cm}$ period hybrid undulator. The theoretical understanding of this unconventional, azimuthally varying field type of RTM has been extended. A comparison of analytically calculated orbit stability with that based on measured data will be presented; orbit calculations using measured field data show the designed performance. Construction and tuning of the $1300 \mathrm{MHz}, 2.2 \mathrm{MV}$ microwave cavity have been completed, and signal level measurements have been performed. The overall assembly of the microtron is nearing completion. At present a vacuum pressure better than $5 \times 10^{-7}$ Torr is achieved.
\end{abstract}

\section{Introduction}

The objective of the Dutch FEL project TEUFEL [1] is to realize an experimental free electron laser for studying the fundamental FEL phenomena, and for contributing to technological developments in this field. The TEUFEL project comprises two phases. In the first phase, $180 \mu \mathrm{m}$ wavelength radiation will be generated in a system consisting of a photocathode linear accelerator delivering high intensity electron pulses at an energy of $6 \mathrm{MeV}$, and an undulator with a periodicity distance of $25 \mathrm{~mm}$ and a $K$-value equal to 1 . An optical cavity will not be employed. In the second phase, the racetrack microtron (RTM), being built at the Eindhoven University, will be incorporated in the system, and the undulator will be housed inside an optical cavity. The RTM is injected from the $6 \mathrm{MeV}$ linac and boosts the energy to $25 \mathrm{MeV}$. Then $10 \mu \mathrm{m}$ radiation will emerge from the undulator.

The choice for the RTM in this project $[2,3]$ was based on the fact that this type of machine inherently provides a small momentum spread and pulse length for the generated electron bunches, and that it is economical, both in construction and in operating costs. However, it remains to be seen experimentally what current the microtron is capable to handle.

In this paper, we elaborate on the orbit dynamic studies, making use of field measurements of the microtron magnets, on the cavity tests, and on a system to be devised for phase and amplitude control of both

\footnotetext{
* Corresponding author.
}

linac and microtron cavity, using the same power source, namely the existing klystron ( $20 \mathrm{MW}$, Thomson TH 2022 C).

\section{Azimuthally varying field}

For cyclotrons, it is well known that simultaneous horizontal and vertical orbit stability as well as isochronism can be achieved in an elegant way by subjecting the beam to an azimuthally varying magnetic field. We have applied similar ideas to our RTM, i.e. we superimposed an azimuthally varying field (AVF) profile on the main average magnetic field of the bending magnets. With such a design, auxiliary focusing elements are no longer needed to obtain stable transverse beam motion.

An extensive canonical treatment of beam dynamics in an AVF-RTM can be found in ref. [4]. The median plane field in the AVF magnet is split into a constant main field and a flutter profile depending only on azimuth. The flutter profile is assumed to be small, so a first order solution for the particle motion has been derived. In the canonical description, we incorporated the following aspects: (i) mirror symmetry of the reference orbit; (ii) arbitrarily shaped AVF profile; (iii) magnet tilt angle to guarantee closed orbits; (iv) drift space; and (v) fringe field lenses at the magnet entrance and exit. It has been shown that tilted magnets are actually an essential requirement for simultaneous horizontal and vertical beam stability.

In the RTM that is under construction at our laboratory, a convenient two-sector hill/valley AVF profile 


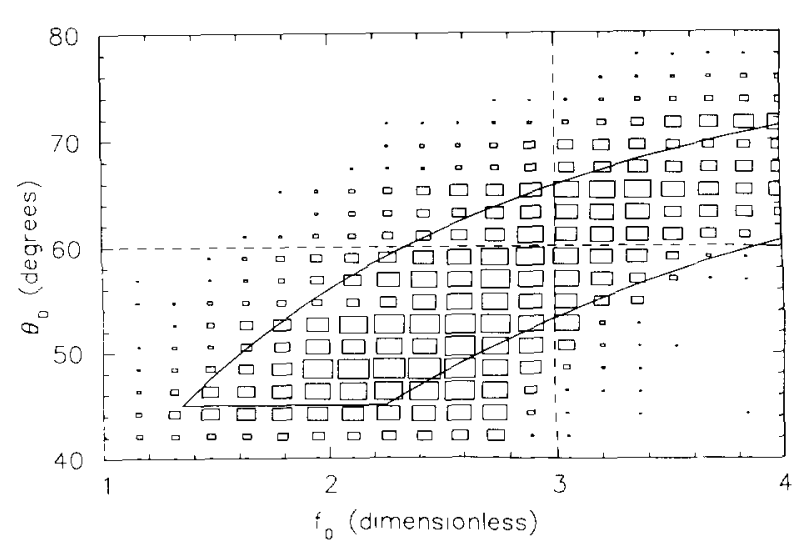

Fig 1. A comparison between numerical results and analytical theory: the drawn curvi-rectangle is the common stability area as predicted by theory; the sizes of the boxes are a figure of merit for the machine acceptance as obtained from numerical calculations.

is used, characterized by $f_{0}$ (relative increase of the induction in the hill with respect to the valley) and $\theta_{0}$ (azimuth of the borderline between valley and hill). By substituting this specific flutter profile into the general stability condition as obtained from the canonical treatment, we obtain a region in the $\left(f_{0}, \theta_{0}\right)$ plane in which stable particle motion in the horizontal and vertical directions is achieved for all relevant values of the beam energy in our machine. The outline of this region is represented by the drawn line in Fig. 1. From numerical calculations (orbit integrations from injection to extraction through computed fieldmaps with all fringe fields taken into account), we calculated the machine acceptance as a function of $f_{0}$ and $\theta_{0}$ as well. The boxes in Fig. 1 show the result, the size of each box being a measure for the machine acceptance. The perfect agreement with the theoretically obtained result is evident.

The best parameter choice inside the stability region turns out to be $f_{0}=0.3$ and $\theta_{0}=60^{\circ}$, i.e. a $30 \%$ higher induction in the hill sector than in the valley. This choicc has already been materialized in our RTM. Magnetic field measurements have been performed to validate the theoretical and numerical results. A special measuring procedure has been used for the study of orbit dynamics: a Hall probe on an $X-Y$ table follows the actual clectron paths by repeatedly using the measured local magnetic field for calculating the new probe position. In Fig. 2, the traces of the horizontal and vertical transfer matrices as a function of path length are shown for one specific beam energy, viz. 16 $\mathrm{MeV}$. The drawn lines in this graph are the result of measurements, the dashed lines of numerical calculations. The difference is very small in both transverse directions. Thus, the excellent agrecment between the- ory, numerical results and measurements has been demonstrated.

\section{The rf accelerating cavity}

Details of the cavity have been given in ref. [5]. Tuning and brazing have now been completed. The measured frequency spectrum around $1300 \mathrm{MHz}$ is shown in Fig. 3. The middle peak corresponds with the $\pi / 2$ accelerating mode. The resonance of this mode at $1300 \mathrm{MHz}$ is achieved at a cavity temperature of $34^{\circ} \mathrm{C}$. The symmetry of the spectrum indicates that the relative tuning of the cells with respect to each other is also satisfactory. The measured quality factor is $Q_{0}=$ 18330. From this we estimate that the corrected shunt impedance is $R_{\varsigma} \approx 14.5 \mathrm{M} \Omega$. The measured generator-cavity coupling coefficient is $\beta=6.7$. Based on these values the required generator power and the reflected power in dependence of the average macropulse beam current can be obtained with formulas given in ref. [6]. Zero reflection is imposed at an average current of $100 \mathrm{~mA}$.

\section{Rf control of the RTM cavity}

Since the beam loading of the injector and the RTM cavity differ substantially, the ratio of if power flowing to both cavities must be adjusted (within $\approx 1$ $\mu \mathrm{s})$ at the moment that the beam is turned on. For this purpose a special control unit may be placed in the waveguide that feeds power to the RTM cavity. This is illustrated in Fig. 4. The control unit will consist of two coupled rf transmission cavities. By detuning these cavities, the power flow at the output can be adjusted.

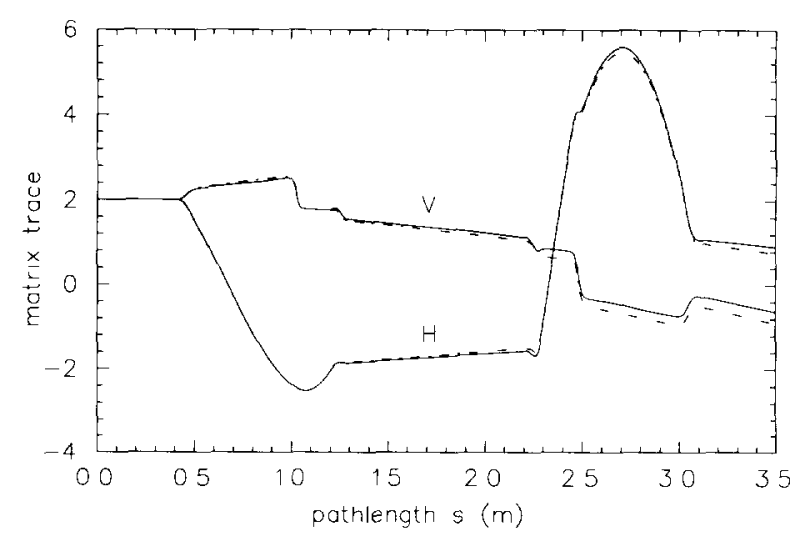

Fig. 2. Matrix traces of horizontal and vertical motion as a function of pathlength at an energy of $16 \mathrm{MeV}$; drawn lines: measurements, dashed lines: numerical results. 


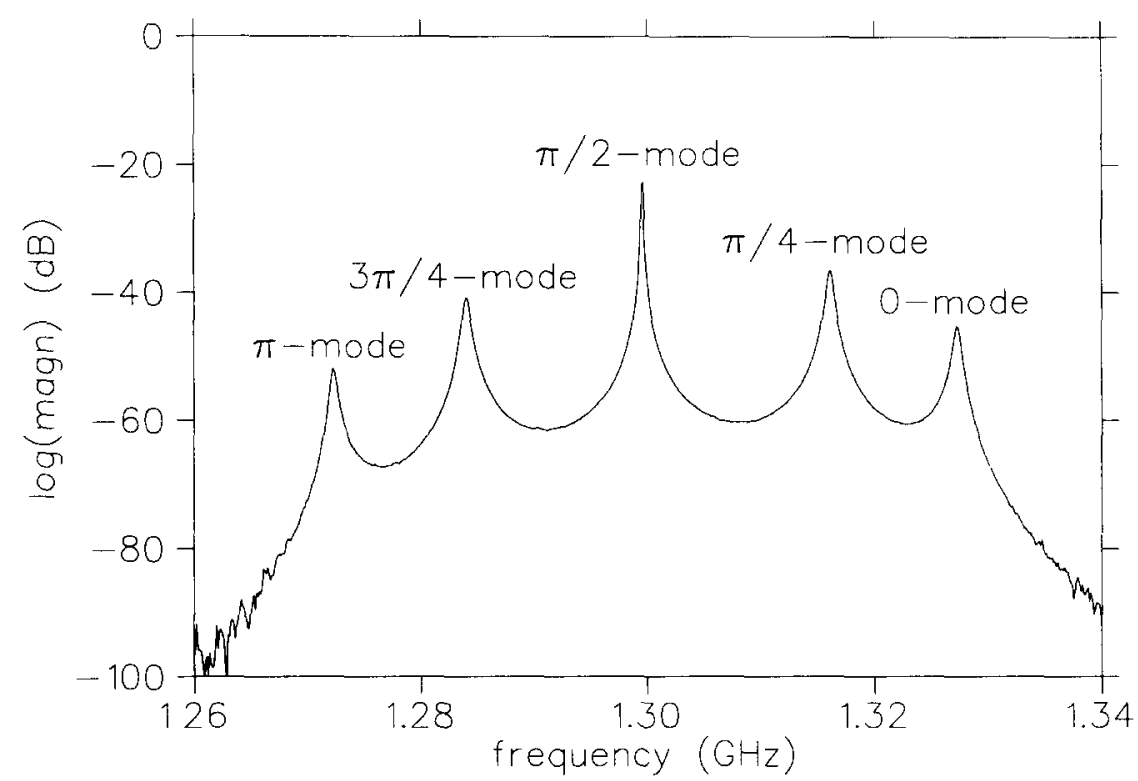

Fig. 3. Frequency spectrum of the RTM cavity near $1300 \mathrm{MHz}$ measured after brazing.

Cavity detuning will be done by loading it with a low energy dc electron beam. A loaded cavity can be modelled with an equivalent circuit as depicted in Fig. 5a [7]. The dc beam loading is taken into account by the impedance $Z_{\mathrm{b}}$ placed parallel to the unloaded cavity circuit. Using this circuit assumes two main approxima- tions namely that the rf cavity voltage is small compared to the beam input energy and further that space charge effects are negligible.

To obtain an estimate of the dc current that is needed, consider the simple case where a uniform beam is injected into a plane parallel gap. For this case

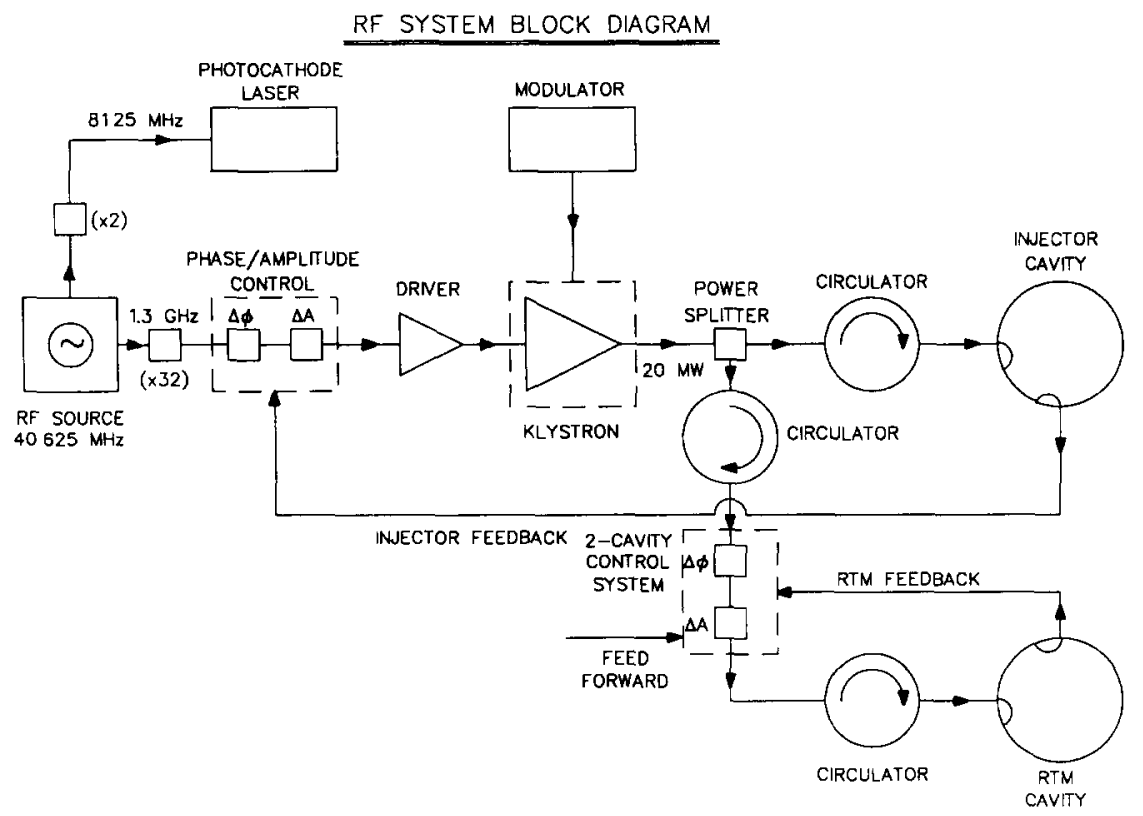

Fig. 4. Block diagram of the TEUFEL rf system. 
a)

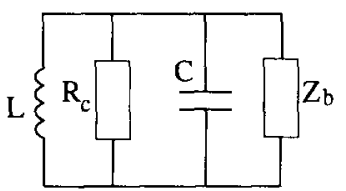

b)

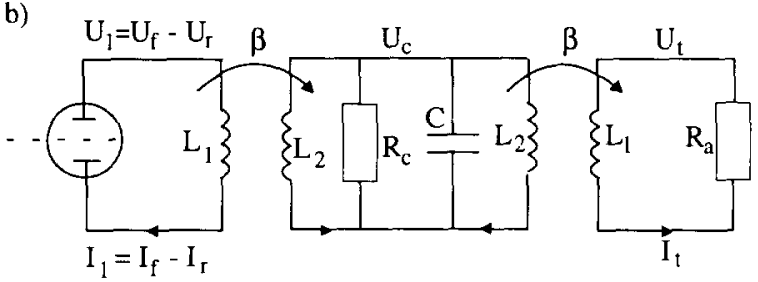

Fig. 5. (a) Equivalent circuit of an isolated if cavity; the impedance $Z_{\mathrm{b}}$ models the loading with a dc electron beam; (b) equivalent circust of a transmission cavity.

the admittance $Y_{\mathrm{b}} \equiv 1 / Z_{\mathrm{b}}$ is given by $Y_{\mathrm{b}}=G_{\mathrm{b}}+\mathrm{j} B_{\mathrm{b}}$ with [7]

$G_{\mathrm{b}}=\frac{I_{0}}{2 V_{0}} \frac{\sin (D / 2)}{D / 2}\left(\frac{\sin (D / 2)}{D / 2}-\cos (D / 2)\right)$,

$B_{\mathrm{b}}=\frac{I_{0}}{2 V_{0}} \frac{\cos (D / 2)}{D / 2}\left(\frac{\sin (D / 2)}{D / 2}-\cos (D / 2)\right)$,

where $I_{0}$ is the dc beam current, $V_{0}$ is the beam input voltage, $D=\omega d / \mathrm{v}_{0}, \omega$ is the rf angular frequency, $d$ is the gap width and $v_{0}$ is the initial electron velocity. The real part of the admittance may give rise to losses and then lowers the effective $Q$-value of the cavity. This is unfavourable, because more detuning would be needed. Therefore it is advisable to design the cavity such that for given frequency $\omega$ and beam velocity $v_{0}$, the real part of the admittance is zero. For the plane parallel gap this is achieved by choosing $D=2 \pi$. Then the imaginary part is given by $B_{\mathrm{b}}=-I_{0} /\left(2 \pi V_{0}\right)$ and this results in a frequency detuning of

$\frac{\Delta \omega}{\omega}=-\frac{R_{\mathrm{c}} B_{\mathrm{b}}}{2 Q_{0}} \approx \frac{R_{\mathrm{c}} I_{0}}{4 \pi Q_{0} V_{0}}$,

where $Q_{0}$ is the unloaded quality factor of the cavity.

To study the effect of detuning we consider the equivalent circuit in Fig. 5b which represents a transmission cavity (see also refs. [8,9]). The rf power flowing towards the cavity is assumed to be generated by a current source with an output impedance $R_{\mathrm{a}}$ equal to the characteristic impedance of the waveguide. The input and output coupling irises of the cavity are represented by ideal transformers with step-up ratio $N$ $=\sqrt{L_{2} / L_{1}}$. At the cavity input there is a forward wave with current $I_{\mathrm{f}}$ and voltage $U_{\mathrm{f}}$ and a reflected wave with current $I_{\mathrm{r}}$ and voltage $U_{\mathrm{r}}$. The output waveguide is terminated with a matched load, so there only is a transmitted wave with current $I_{\mathrm{t}}$ and voltage $U_{\mathrm{t}}$. The transmitted and reflected voltage waves and also the voltage in the cavity are related to the forward generator current by transfer impedances which are found by solving the circuit equations:

$Z_{\mathrm{t}}=\frac{U_{\mathrm{t}}}{I_{\mathrm{f}}}=\frac{2 \beta R_{\mathrm{d}}}{1+2 \beta} \cos \psi \mathrm{e}^{\lrcorner \psi}$,

$Z_{\mathrm{c}}=\frac{U_{\mathrm{c}}}{I_{\mathrm{f}}}=N Z_{\mathrm{t}}$,

$Z_{\mathrm{r}}=\frac{U_{\mathrm{r}}}{I_{\mathrm{f}}}=Z_{\mathrm{t}}-R_{\mathrm{a}}$.

Here, $\beta=\left(R_{\mathrm{c}} L_{1}\right) /\left(R_{\mathrm{a}} L_{2}\right)$ is the cavity coupling coefficient and $\psi$ is the cavity detuning angle defined by

$\tan \psi=-\frac{2 Q_{0}}{1+2 \beta} \frac{\Delta \omega}{\omega}$.

As a remark we note that the circuit obeys energy conservation, i.e. from Eqs. (4) it easily follows that the forward power $P_{\mathrm{f}}=R_{\mathrm{a}} I_{\mathrm{f}}^{2} / 2$ equals the sum of the reflected power $P_{\mathrm{r}}=U_{\mathrm{r}}^{2} / 2 R_{\mathrm{a}}$ plus the transmitted power $P_{\mathrm{t}}=U_{\mathrm{t}}^{2} / 2 R_{\mathrm{a}}$ plus the dissipated power $P_{\mathrm{c}}=$ $U_{\mathrm{c}}^{2} / 2 R_{\mathrm{c}}$.

To have a significant detuning, the angle $\psi$ should be close to $45^{\circ}$ because then there would be a reduction in transmitted power with a factor of 2 . From Eqs. (3) and (5) it then follows that

$I_{0} / V_{0} \approx 2 \pi(1+2 \beta) / R_{\mathrm{c}}$.

If we assume an initial beam voltage $V_{0}=10 \mathrm{kV}$, a coupling coefficient $\beta=5$ and a cavity shunt impedance $R_{\mathrm{S}}=2 R_{\mathrm{c}}=10 \mathrm{M} \Omega$, then the required current becomes $I_{0}=150 \mathrm{~mA}$. However, since we want to be able to adjust the angle $\psi$ into positive as well as negative directions, also an off-set of $150 \mathrm{~mA}$ is needed so that the total maximum current would be $300 \mathrm{~mA}$, corresponding with a beam power of approximately $3 \mathrm{~kW}$.

Since both the amplitude and phase of the transmitted wave must be independently adjustable, two transmission cavities placed in series and connected by a waveguide are needed. In this waveguide there will be a forward wave (as transmitted from cavity 1) and a backward wave (as reflected from cavity 2). It is easily shown that an isolator must be placed between the two cavities in order to absorb the reflected wave; otherwise the system would behave like just one cavity with only one free parameter. For this system the total transmission impedance is given by

$Z_{\mathrm{t}}=\frac{4 \beta^{2}}{(1+2 \beta)^{2}} R_{\mathrm{a}} \cos \psi_{1} \cos \psi_{2} \mathrm{e}^{\mathrm{\jmath}\left(\psi_{1}+\psi_{2}\right)}$.

If the phase $\psi=\psi_{1}+\psi_{2}$ of the transmitted wave is chosen, then the amplitude can still be varied by choosing proper combinations of $\psi_{1}$ and $\psi_{2}$. Physically important is the maximum power which is available at the 
output, for a given input power and for a certain choice of $\psi$. This maximum power range, normalized with respect to the input power, is given by

$$
p=\frac{16 \beta^{4}}{(1+2 \beta)^{4}} \cos ^{4} \frac{\psi}{2} \text {. }
$$

From this equation it follows that $\beta$ should not be too small in order to have efficient power transfer. For $\beta=5$ the maximum power transfer $(\psi=0)$ is $68 \%$. On the other hand, if $\beta$ increases, the current needed for detuning increases proportionally as follows from Eq. (6). In practice a compromise must be found which takes into account the power that is available at the input, the maximum power that is needed at the output and the dc beam current that can be achieved.

\section{References}

[1] G.J. Ernst, W.J. Witteman, J.W.J. Verschuur, E.H. Haselhoff, R.F.X.A.M. Mols, A.F.M. Bouman, J.I.M Botman, H.L. Hagedoorn, J.L. Delhez and W.J.G.M. Kleeven, Nucl. Instr. and Meth. A 318 (1992) 173.
[2] J.I M. Botman, J.L. Delhez, G.A. Webers, H L. Hagedoorn, W.J.G.M. Kleeven, C.J. Timmermans, G J. Ernst, J.W.J. Verschuur, W.J Witteman and E.H. Haselhoft, Nucl. Instr. and Meth. A 304 (1991) 192

[3] J.I.M. Botman, G.A Webers, J.L. Delhez, C.J Timmermans, M.E.H.J Theeuwen, W.J.G.M. Kleeven, H.L. Hagedoorn, G.J. Ernst, J.W.J. Verschuur and W J Witteman, Nucl. Instr. and Meth. A 318 (1992) 358.

[4] J.L. Delhez and W.J.G.M. Kleeven, Part. Acc. 42 (1993) 101.

[5] W.J.G.M Kleeven, M.E.H.J. Theeuwen, M.H.M. Knoben. A.J. Moerdık, J.I.M. Botman, J.A. van der Heıde. C.J. Timmermans and H L. Hagedoorn, Nucl. Instr. and Meth. B 68 (1992) 87.

[6] W.J.G.M Kleeven, A.J. Moerdijk, J.I.M. Botman, J.L. Delhez, H.L. Hagedoorn, J.A. van der Hejde, M.H.M Knoben, M.E.H.J. Theeuwen, C.J. Timmermans and G.A. Webers, Proc. 3rd Europ. Particle Accelerator Conf (EPAC), Berlin, March 24-28, 1992, p. 813.

[7] M. Chodorow and C. Susskind, Fundamentals of M1crowave Electronics (McGraw-Hill, New York. 1964).

[8] P.B. Wilson, in: Physics of High Energy Particle Accelerators, AIP Conf. Proc No. 87 (1982)

[9] A Gamp, CERN School on RF Engineerıng for Particle Accelerators, CERN 92-03 (1992) Vol. II 